\title{
Lipid mediator lipoxin A4 and its analog BML-111 exert antitumor effects in melanoma
}

\author{
Yu Du ${ }^{1,2 \#}$, Jianing Yang ${ }^{2,3 \#}$, Tangfeng Su ${ }^{4 \#}$, Zhu Shen ${ }^{2,3}$, Juan $\mathrm{Li}^{2,3}$ \\ ${ }^{1}$ Department of Anesthesiology, Sichuan Provincial People's Hospital, University of Electronic and Technology of China, Chengdu, China; ${ }^{2}$ Chinese \\ Academy of Sciences Sichuan Translational Medicine Research Hospital, Chengdu, China; ${ }^{3}$ Department of Dermatology, Sichuan Provincial People's \\ Hospital, University of Electronic and Technology of China, Chengdu, China; ${ }^{4}$ Department of Pediatrics, Tongji Hospital, Tongji Medical College, \\ Huazhong University of Science and Technology, Wuhan, China \\ Contributions: (I) Conception and design: J Li, Z Shen; (II) Administrative support: None; (III) Provision of study materials or patients: J Yang; \\ (IV) Collection and assembly of data: Y Du; (V) Data analysis and interpretation: T Su; (VI) Manuscript writing: All authors; (VII) Final approval of \\ manuscript: All authors. \\ \#These authors contributed equally to this work. \\ Correspondence to: Juan Li. Department of Dermatology, Sichuan Provincial People's Hospital, University of Electronic and Technology of China, No. \\ 32 West Second Section First Ring Road, Chengdu, China. Email: eyre09@163.com.
}

\begin{abstract}
Background: LipoxinA4 (LXA4) is an anti-inflammatory lipid mediator which was recently proposed to have antitumor potential. However, the therapeutic effect of LXA4 in melanoma is still unclear. This work aimed to investigate the function of LXA4 and its analog in melanoma invasion through in vivo and in vitro experiments.
\end{abstract}

Methods: The expression of the LXA4 receptor (ALXR) was detected in melanoma tissues and A375 human melanoma cells, using benign melanocytic nevi tissues and human melanocytes as negative controls, respectively. The invasive and apoptotic abilities of A375 cells in the presence or absence of LXA4 were examined by cell invasion assay and flow cytometric analysis. Finally, mice melanoma models were established, and the antitumor effects of BML-111 [5(S), 6(R)-7-trihydroxymethyl heptanoate], an agonist of ALXR, were examined in vivo.

Results: ALXR was abundantly expressed in human melanoma tissues. The ALXR messenger RNA (mRNA) and protein expression levels were higher in A375 melanoma cells than in the controls $(\mathrm{P}<0.05)$. LXA4 could significantly attenuate the invasion ability of A375 cells $(\mathrm{P}<0.05)$. This trend was further enhanced by BML-111, which tended to control the tumor development in A375 melanoma models.

Conclusions: LXA4 and its analog BML-111 exert antitumor effects in vivo and in vitro, and may be potential therapeutic options for patients with invasive melanoma.

Keywords: Lipoxin A4 (LXA4); BML-111; melanoma

Submitted Mar 22, 2021. Accepted for publication May 08, 2021.

doi: 10.21037/atm-21-1873

View this article at: http://dx.doi.org/10.21037/atm-21-1873

\section{Introduction}

Melanomas are an extremely aggressive form of lifethreatening cancer, with an incidence that has shown a dramatic increase over the past few years (1). Although melanomas are curable with surgical excision when diagnosed early, accurate assessment of disease progression is critical. Melanomas are associated with a high risk of mortality, thus, it is vital to explore their underlying molecular mechanisms to aid the development of new therapies.

LXA4 is an endogenous eicosanoid (2-4), which serves as the 'stop signal' for inflammation and binds to the highaffinity G protein-coupled ALXR to generate signals, via which potent inflammation-alleviating effects are 
exerted. Although the antitumorigenic effects of LXA4 have been observed in different tumor models (5-7), their underlying mechanism remains unclear. LXA4 and its analogs were found to potently inhibit cell proliferation in a lung adenocarcinoma cell line (8), and in umbilical vein endothelial cells stimulated with vascular endothelial growth factor (9). Thus, we speculated that LXA4 could modulate melanoma cell proliferation and migration by binding to ALXR.

In this work, we sought to evidence the antitumor effects of LXA4 and its analog BML-111 in A375 melanoma cell and murine melanoma models. Our findings testified that LXA4 may possess an inherent ability to inhibit melanoma growth.

We present the following article in accordance with the ARRIVE reporting checklist (available at http://dx.doi. org/10.21037/atm-21-1873).

\section{Methods}

\section{Tissue specimens}

Tissue samples were donated by patients with melanoma who received treatment in the Department of Dermatology, Sichuan Provincial People's Hospital, University of Electronic Science and Technology of China. All procedures performed in this study involving human participants were in accordance with the Declaration of Helsinki (as revised in 2013). The study was approved by the Ethics Committee of Sichuan Provincial People's Hospital, University of Electronic Science and Technology of China (No.: Sichuan Provincial People's Hospital-D-2012-158) and informed consent was taken from all the patients.

\section{Immunobistochemistry}

Employing primary ALXR antibodies (Santa Cruz Biotechnology, Santa Cruz, CA, U.S.A.), we performed immunohistochemical staining to detect the expression of ALXR in paraffin dermal tissue sections. Brown staining indicated positivity. In all experiments, controls for immune-specificity were included and the primary antibody was replaced with phosphate-buffered saline (PBS).

\section{Cell viability assay}

Cell viability was examined by seeding cells on a 96-well plate with 5,000 cells/well. A 3-(4,5-dimethyl-2-thiazolyl)-
2,5-diphenyl-2H-tetrazolium bromide (MTT) assay kit was used to measure cell viability according to the manufacturer's protocol. The absorbance was measured at a reference wavelength of $570 \mathrm{~nm}$.

\section{Quantitative real-time PCR}

The extraction of total RNA was performed with Life Technologies TRIzol reagent. The concentration and quality of the RNA were measured using a Nanodrop Spectrophotometer (Thermo Fisher Scientific, Rockford, IL, USA). To synthesize complementary DNA, reverse transcription was carried out on $1 \mu \mathrm{g}$ of RNA using the Revert Aid First Strand Synthesis kit (Fermentas, Glen Burnie, MD, USA). SYBR ${ }^{\circledR}$ Premix Ex Taq (Takara Bio Inc., Shiga, Japan) was used for quantitative realtime PCR (qRT-PCR) analysis, in adherence with the protocol supplied by the manufacturer. Each sample was normalized and analyzed in triplicate. $\beta$-actin served as an internal control. Sequence of primers used for qRTPCR. ALX: 5'-CTGACAGCCAGAGGGATTA-3', 5'-TCTCTTTGAGCCAGACGGT - 3 '; $\beta$-actin: 5'-AAGGCCAACCGCGAGAA-3'，5'-CCTCGTAG ATGGGCACA-3'; ALX-siRNA: 5'-UCUUCAUAUU CAUUCAGAGGA-3', 5 '-CUCUGAAUGAAUAUG AAGAAG-3'.

\section{Western blot}

RIPA lysis buffer was used for cell and tissue protein extraction, and the BCA Protein Assay kit (Pierce, Rockford, IL, USA) was used to measure the concentrations of proteins. We separated identical quantities of protein by sodium dodecyl sulfate-polyacrylamide gel electrophoresis (SDS-PAGE) before transferring them to polyvinylidene fluoride (PVDF) membranes (Millipore, Bedford, MA). The membranes were subjected to incubation, first in a blocking buffer, then with anti-ALXR $(1: 1,000)$ monoclonal antibodies (Santa Cruz Biotechnology, Santa Cruz, CA, USA). Protein expression was normalized against $\beta$-actin expression (Santa Cruz Biotechnology, Santa Cruz, CA, USA). An imaging densitometer was employed for quantitative blotting image analysis.

\section{Cell invasion assay}

To assess the invasion abilities of A375 cells, we carried out a chamber-based cell invasion assay following the kit protocol. 
Invasive cells were counted, and 10 random fields on each membrane were photographed at $200 \times$ magnification. The values were the averages of 3 experiments.

\section{Detection of apoptotic cells}

Cells were collected and then subjected to apoptotic analysis. Cells were labeled with Annexin-V-FITC and propidium iodide for 15 minutes in a darkroom. Using the FACScan flow cytometer, the percentage of apoptotic cells was calculated.

\section{Cell lines and mice}

Melanocytes and A375 cells were kindly supplied by Prof. Chen (Union Hospital, Tongji Medical College, Huazhong University of Science and Technology, China). Cell culture was performed in Dulbecco's Modified Eagle Medium containing 10\% fetal bovine serum (Gibco, Grand Island, NY, USA) under the conditions of $37{ }^{\circ} \mathrm{C}$ with $5 \% \mathrm{CO}_{2}$. Female BALB/c nude mice, aged 4-6 weeks, purchased from the animal research center of Sichuan Provincial People's Hospital, University of Electronic Science and Technology of China, were used for the model. Each experiment was conducted in adherence with the National Institutes of Health Guide for the Care and Use of Laboratory Animals, and was approved by the ethics committee of Sichuan Provincial People's Hospital, University of Electronic Science and Technology of China (NO.: Sichuan Provincial People's Hospital-D-2012-51).

\section{Animal experiments}

The BALB/c-nu/nu mice were administered a subcutaneous injection of $1 \times 10^{6} \mathrm{~A} 375$ cells. We administered the mice with intraperitoneal injections of BML-111 $(1 \mathrm{mg} / \mathrm{kg})$, LXA4 $(10 \mu \mathrm{g} / \mathrm{kg})$, or PBS at 2-day intervals, until the tumors exceeded $5 \mathrm{~mm}$ in size. The general conditions of the mice, including their activity and weight were observed daily. To avoid deviation, the experimenter and the feeder have their own responsibilities.

\section{Histological examination}

Melanoma samples were obtained from the mice after the completion of the experiment. The skin specimens were subjected to routine hematoxylin and eosin staining. For the avoidance of bias, the study design included single-blinding of the histologist to the mouse treatment groups.

\section{Statistical analysis}

Data analyses were conducted using SPSS version 22.0 software (SPSS Inc., Chicago, IL, USA). The data in this study were presented as means \pm standard error of mean (SEM). Differences with $\mathrm{P}<0.05$ were deemed to have statistical significance. Independent $t$ tests or MannWhitney $U$ tests were applied to check the significance level of differences. Each experiment was performed for a minimum of 3 times.

\section{Results}

\section{ALXR expression is increased in melanoma tissues}

LXA4 functions through activation of the ALXR G-proteincoupled receptor. To investigate the ALXR expression in melanoma, paraffin sections were handled as detailed in the Materials and Methods. ALXR expression was detected at low to moderate levels in benign melanocytic nevi tissue, but a strong expression was seen in melanoma tissue (Figure 1A). Meanwhile, the mRNA and protein level of ALXR in melanoma tissues were examined (Figure 1B,C). We also comparatively analyzed the ALXR mRNA and protein profiles in A375 melanoma cells and melanocytes. As shown in Figure 2A,B, real-time RT-PCR and western blotting showed increased ALXR mRNA and protein expression in A375 cells compared to melanocytes.

\section{LXA4 inbibits A375 cell proliferation}

We studied the effects of LXA4 in melanoma by transfecting A375 cells with LXA4 and performing gain-of-function analyses. As shown in Figure 2C, compared to the control cells, overexpression of LXA4 dramatically decreased the A375 cell growth rate. We transfected A375 cells with ALXR small interfering RNA (siRNA) using Lipofectamine 2000 (Invitrogen, USA). In contrast, cell proliferation was markedly increased after transfection, suggesting that LXA4 suppressed the proliferation of melanoma cells.

\section{Effects of LXA4 on cell invasion and apoptosis}

Next, we examined the biological functions of LXA4 in cell invasiveness and apoptosis by performing an invasion assay and flow cytometry, respectively. In comparison to the 
A

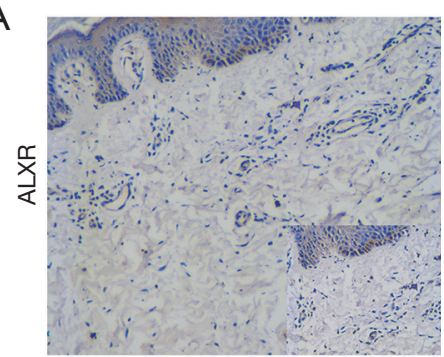

Control

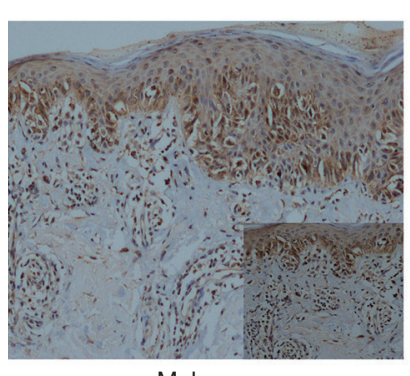

Melanoma
C
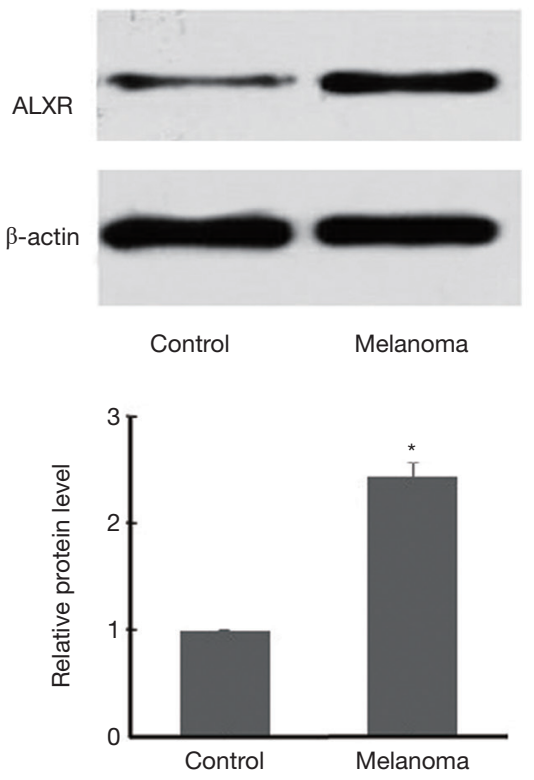

Figure 1 Increased localization of ALXR in melanoma tissues. (A) Paraffin sections of benign melanocytic nevi and melanoma were prepared and labelled with antibodies specific for ALXR using immunohistochemical staining (scale bars: $100 \mu \mathrm{m}$ ). In the panels, the inset shows the same tissue viewed under a microscope (scale bars: $200 \mu \mathrm{m}$ ). (B) ALXR messenger RNA (mRNA) expression in melanoma tissues. ALXR mRNA expression was assessed using RT-PCR. (C) ALXR protein expression in melanoma tissues. ALXR protein expression were assessed using western blot. The Mann-Whitney $\mathrm{U}$ test was applied for statistical analysis; $\mathrm{n}=5$, values are the mean $\pm \mathrm{SEM}$. ${ }^{*} \mathrm{P}<0.05$. ALXR, LipoxinA4 receptor; RT-PCR, real-time polymerase chain reaction; SEM, standard error of the mean.

A

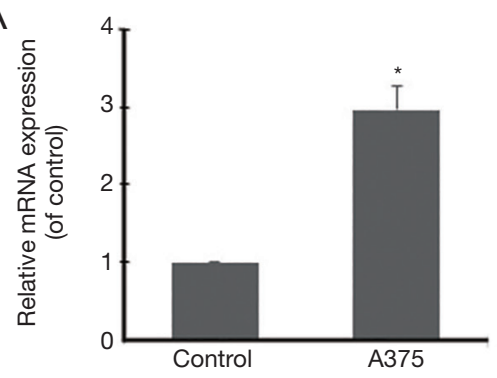

C

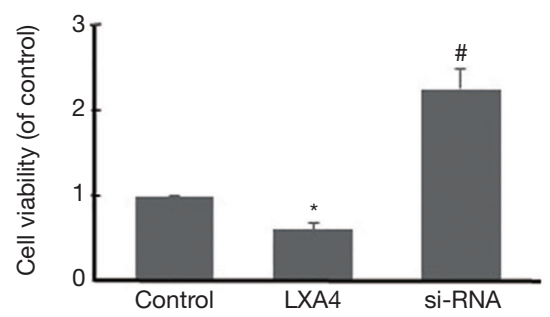

B
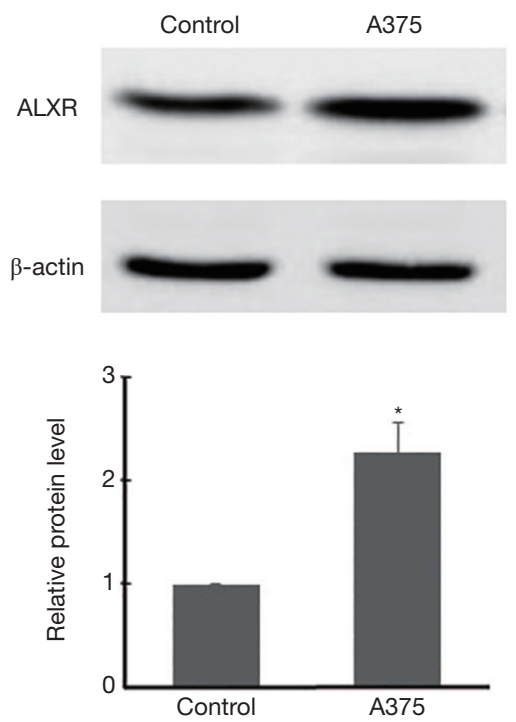

Figure 2 ALXR mRNA and protein expression in A375 melanoma cells and melanocytes. ALXR mRNA expression (A), ALXR protein expression (B) and cell proliferation (C) were assessed using RT-PCR, western blot and MTT. The Mann-Whitney U test was applied for statistical analysis; $\mathrm{n}=5$, values are the mean $\pm \mathrm{SEM}$. ${ }^{*} \mathrm{P}<0.05,{ }^{*} \mathrm{P}<0.01$. ALXR, LipoxinA4 receptor; mRNA, messenger RNA; RT-PCR, realtime polymerase chain reaction; MTT, 3-(4,5-dimethyl-2-thiazolyl)-2,5-diphenyl-2H-tetrazolium bromide; SEM, standard error of the mean. 
A

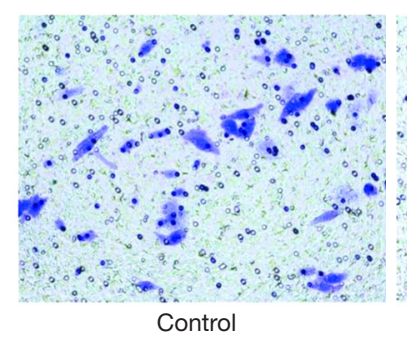

B

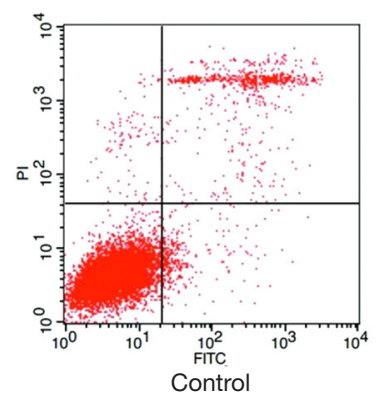

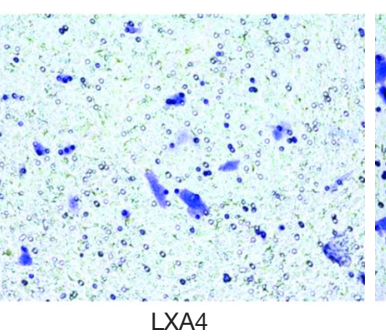
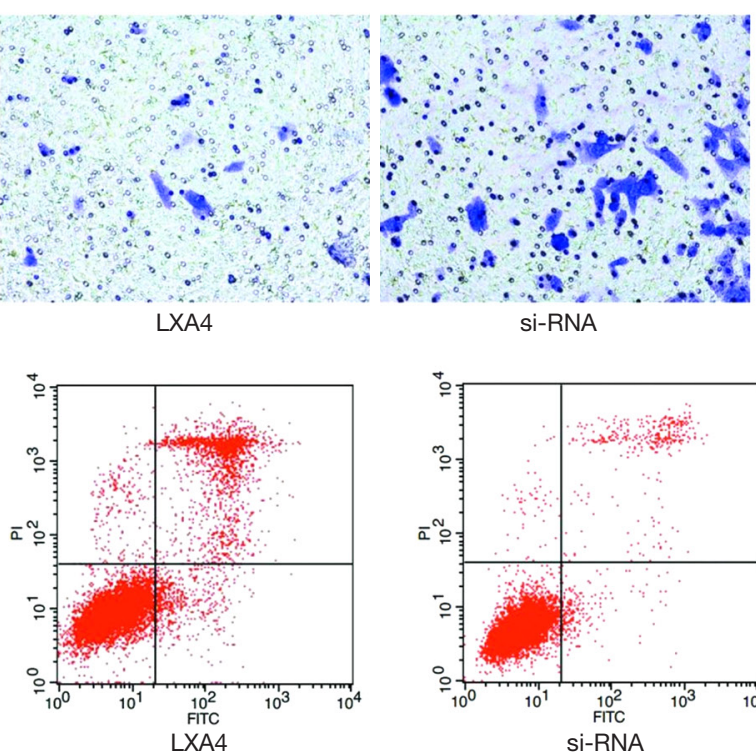

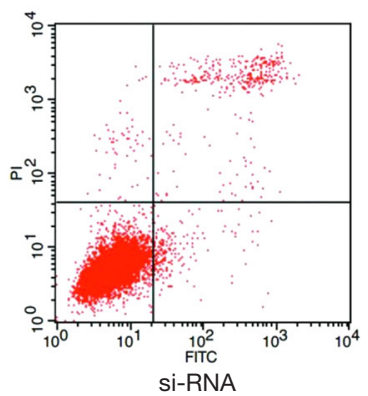

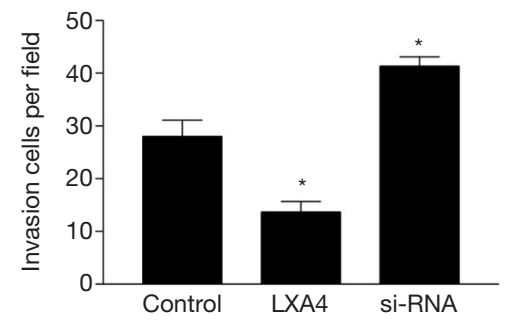

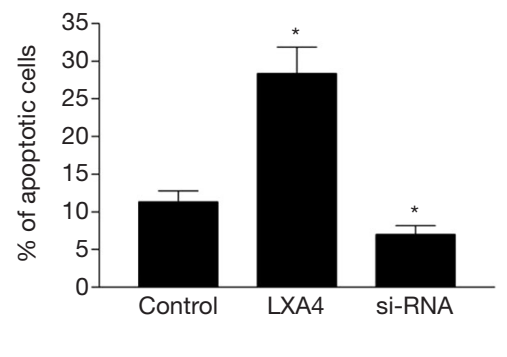

Figure 3 Effects of LXA4 on cell invasion and apoptosis. (A) Photomicrographs showing a Matrigel Boyden chamber with fewer colonies in A375 cells overexpressing LXA4 compared to the controls using crystal violet (0.1\%) staining (scale bars: $200 \mu \mathrm{m}$ ). Invading A375 cells were normalized to the cell mass on the bottom sides of the membrane ( $n=3$, mean $\pm S E M)$. ${ }^{*} \mathrm{P}<0.01$. (B) Flow cytometric analysis show that silencing of LXA4 receptor protects A375 cells from apoptosis. ${ }^{*} \mathrm{P}<0.05$. LXA4, LipoxinA4; SEM, standard error of the mean.

control cells, LXA4 overexpression drastically weakened the invasive ability of A375 cells. Consistent with the invasion experiment results, apoptosis data analysis showed that LXA4 overexpression induced a significant increase in apoptotic death. In contrast, the silencing of ALXR expression strengthened the invasive capability of A375 cells, seemingly protecting them from apoptosis (Figure 3). These results suggested that LXA4 significantly promoted apoptosis and inhibited the invasion of A375 cells.

\section{Antitumor effects of LXA4 and BML-111 in the melanoma mouse model}

Finally, we assessed the antitumor effects of LXA4 by establishing melanoma models in mice. After subcutaneous injection of A375 tumor cells $\left(1 \times 10^{6}\right)$, the model mice were treated until their tumors exceeded $5 \mathrm{~mm}$ in size. Tumorbearing mice (24 mice) were administered with BML111 (8 mice), LXA4 (8 mice), or PBS (8 mice) once every 2 days. As shown in Figure $4 A$, the tumor size in BML111 group was apparently small compared to the control. BML-111 administration significantly inhibited melanoma tumor growth compared with the controls (Figure 4B). Furthermore, hematoxylin and eosin staining revealed that BML-111 destroyed the tumor tissues and reduced the amount of melanoma cells in the tissue (Figure 4C). To clarify whether LXA4 exerted antitumor effects in the melanoma models, we directly subcutaneously injected LXA4 into A375 tumor-bearing mice. No effect on tumor growth was observed after treatment with LXA4 (data not shown), which may undergo rapid conversion to a lipid with biological inactivity and reduced potential as an antiinflammatory mediator.

\section{Discussion}

There is strong evidence that inflammation and angiogenesis share a close connection in a variety of conditions, such as cancer $(10,11)$. Inflammation and chronic infection are related to tumorigenesis in 1 in 5 cancer cases worldwide (12). The risk of developing cancer is elevated for individuals suffering chronic inflammatory diseases $(13,14)$, which suggests inflammation to be, at least partially, implicated in cancer development. Thus, pinpointing the inflammationrelated pathways which have involvement in cancers would aid in the development of new anticancer therapies.

Endogenous anti-inflammatory lipid mediators, like lipoxins, have recently been administered as a 

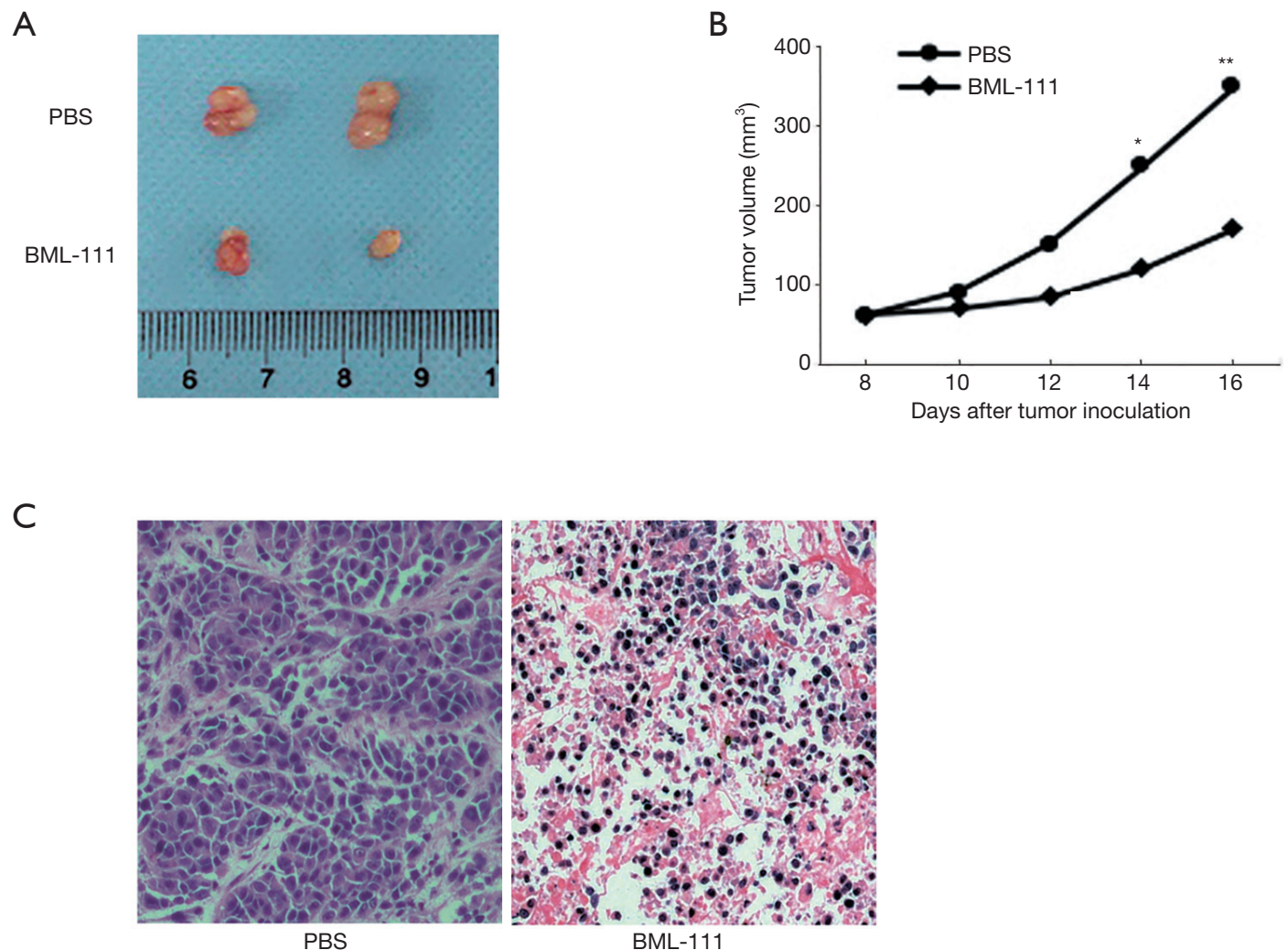

Figure 4 BML-111 inhibits tumor growth in mice. (A) The tumor size of nude mice. (B) BML-111 suppressed tumor growth in mice. A tumor growth assay was performed, and the results of 3 replicates were totaled. ${ }^{*} \mathrm{P}<0.05 ;{ }^{* *} \mathrm{P}<0.01$. (C) Hematoxylin and eosin (H\&E) staining of melanoma samples. Mice were inoculated with $1 \times 10^{6}$ A375 tumor cells and were treated until the tumor size exceeded 5 mm. BML-111 (1 mg/kg) was injected intraperitoneally into the mice every 2 days. At day 16, the mice were euthanized, and their tumors were surgically removed for H\&E staining. Scale bars: $200 \mu \mathrm{m}$. H\&E, Hematoxylin and eosin; BML-111: 5(S),6(R)-7-trihydroxymethyl heptanoate.

new therapeutic strategy for cancers (15). Lipoxins are endogenous eicosanoids produced by 5 - and 15-lipoxygenases or by 5- and 12-lipoxygenases. LXA4, a principle lipoxin, is generated from arachidonic acid which also generates prostaglandins and leukotrienes possessing tumor-promoting effects. Compared with prostaglandins and leukotrienes, LXA4 possesses inherent antitumor properties (16), and it can reduce cell proliferation, inhibit tumor cell invasion, and tumor growth $(5,17)$. Our current work firstly identified that the ALXR levels were increased in MM tissue samples and A375 melanoma cells. Then, by performing a series in vitro and in vivo experiments, we demonstrated that LXA4 and its analog BML-111 contribute greatly to the inhibition of melanoma cell invasion.

In premalignant lesions, LXA4 can block cancer development through the attenuation of chronic inflammation, which can be influenced by LXA4 independent of its anti-inflammation function $(5,18)$. As evidenced in previous researches, by downregulating intracellular reactive oxygen species, LXA4 can suppress the invasive activity of pancreatic cancer cells (19-21). ATL-1 [15-epi-16-(parafluoro)-phenoxy-lipoxin A4], a synthetic analog of lipoxin, can modulate tumor cell transendothelial migration, and acts as a modulator of vascular endothelial growth factor-induced vascular permeability (22). Recent research has evidenced that LXA4 targets immunosuppressive regulatory B cells to serve as an upregulator of antitumor immunity (16). Thus, our followup works aim to explore the underlying mechanisms of LXA4 involved in melanoma.

In summary, the present study showed that LXA4 can attenuate A375 cell invasion. Furthermore, BML-111 reduces the amount of cancer cells in A375 melanoma tissue from mice. Our observations suggest that LXA4 and 
its analog may serve as novel agents to prevent or control cancer development.

\section{Acknowledgments}

Funding: This study was supported by the National Natural Science Foundation of China (NSFC) (No. 81301372).

\section{Footnote}

Reporting Checklist: The authors have completed the ARRIVE reporting checklist. Available at http://dx.doi. org/10.21037/atm-21-1873

Data Sharing Statement: Available at http://dx.doi. org/10.21037/atm-21-1873

Conflicts of Interest: All authors have completed the ICMJE uniform disclosure form (available at http://dx.doi. org/10.21037/atm-21-1873). The authors have no conflicts of interest to declare.

Ethical Statement: The authors are accountable for all aspects of the work in ensuring that questions related to the accuracy or integrity of any part of the work are appropriately investigated and resolved. All procedures performed in this study involving human participants were in accordance with the Declaration of Helsinki (as revised in 2013). The study was approved by the Ethics Committee of Sichuan Provincial People's Hospital, University of Electronic Science and Technology of China (NO.: Sichuan Provincial People's Hospital-D-2012-158) and informed consent was taken from all the patients. Animal experiments were performed under a project license (NO.: Sichuan Provincial People's Hospital-D-2012-51) granted by the Ethics Committee of Sichuan Provincial People's Hospital, University of Electronic Science and Technology of China, in compliance with the National Institutes of Health Guide for the Care and Use of Laboratory Animals.

Open Access Statement: This is an Open Access article distributed in accordance with the Creative Commons Attribution-NonCommercial-NoDerivs 4.0 International License (CC BY-NC-ND 4.0), which permits the noncommercial replication and distribution of the article with the strict proviso that no changes or edits are made and the original work is properly cited (including links to both the formal publication through the relevant DOI and the license).
See: https://creativecommons.org/licenses/by-nc-nd/4.0/.

\section{References}

1. Houghton AN, Polsky D. Focus on melanoma. Cancer Cell 2002;2:275-8.

2. József L, Zouki C, Petasis NA, et al. Lipoxin A4 and aspirin-triggered 15-epi-lipoxin A4 inhibit peroxynitrite formation, NF-kappa B and AP-1 activation, and IL-8 gene expression in human leukocytes. Proc Natl Acad Sci U S A 2002;99:13266-71.

3. Sodin-Semrl S, Taddeo B, Tseng D, et al. Lipoxin A4 inhibits IL-1 beta-induced IL-6, IL-8, and matrix metalloproteinase- 3 production in human synovial fibroblasts and enhances synthesis of tissue inhibitors of metalloproteinases. J Immunol 2000;164:2660-6.

4. Claria J, Lee MH, Serhan CN. Aspirin-triggered lipoxins (15-epi-LX) are generated by the human lung adenocarcinoma cell line (A549)-neutrophil interactions and are potent inhibitors of cell proliferation. Mol Med 1996;2:583-96.

5. Wu SH, Lu C, Dong L, et al. Lipoxin A4 inhibits TNFalpha-induced production of interleukins and proliferation of rat mesangial cells. Kidney Int 2005;68:35-46.

6. Chen $\mathrm{Y}, \mathrm{Hao} \mathrm{H}, \mathrm{He} \mathrm{S}$, et al. Lipoxin $\mathrm{A} 4$ and its analogue suppress the tumor growth of transplanted $\mathrm{H} 22$ in mice: the role of antiangiogenesis. Mol Cancer Ther 2010;9:2164-74.

7. Zhang B, Jia H, Liu J, et al. Depletion of regulatory T cells facilitates growth of established tumors: a mechanism involving the regulation of myeloid-derived suppressor cells by lipoxin A4. J Immunol 2010;185:7199-206.

8. Clària J, Lee MH, Serhan CN. Aspirin-triggered lipoxins (15-epi-LX) are generated by the human lung adenocarcinoma cell line (A549)-neutrophil interactions and are potent inhibitors of cell proliferation. Mol Med 1996;2:583-96.

9. Fierro IM, Kutok JL, Serhan CN. Novel lipid mediator regulators of endothelial cell proliferation and migration: aspirin-triggered-15R-lipoxin A(4) and lipoxin A(4). J Pharmacol Exp Ther 2002;300:385-92.

10. Hanahan D, Weinberg RA. Hallmarks of cancer: the next generation. Cell 2011;144:646-74.

11. Mantovani A, Allavena P, Sica A, et al. Cancer-related inflammation. Nature 2008;454:436-44.

12. Balkwill F, Mantovani A. Cancer and inflammation: implications for pharmacology and therapeutics. Clin Pharmacol Ther 2010;87:401-6. 


\section{Page 8 of 8}

13. Coussens LM, Werb Z. Inflammation and cancer. Nature 2002;420:860-7.

14. Vakkila J, Lotze MT. Inflammation and necrosis promote tumour growth. Nat Rev Immunol 2004;4:641-8.

15. Greene ER, Huang S, Serhan CN, et al. Regulation of inflammation in cancer by eicosanoids. Prostaglandins Other Lipid Mediat 2011;96:27-36.

16. Wang Z, Cheng Q, Tang K, et al. Lipid mediator lipoxin A4 inhibits tumor growth by targeting IL-10-producing regulatory B (Breg) cells. Cancer Lett 2015;364:118-24.

17. Zhou XY, Li YS, Wu P, et al. Lipoxin A(4) inhibited hepatocyte growth fac-tor-induced invasion of human hepatoma cells. Hepatol Res 2009;39:921-30.

18. Hao H, Liu M, Wu P, et al. Lipoxin A4 and its analog suppress hepatocellular car-cinoma via remodeling tumor microenvironment. Cancer Lett 2011;309:85-94.

19. Börgeson E, Lönn J, Bergström I, et al. Lipoxin A4 inhibits porphyromonas gingi-valis-induced aggregation

Cite this article as: Du Y, Yang J, Su T, Shen Z, Li J. Lipid mediator lipoxin A4 and its analog BML-111 exert antitumor effects in melanoma. Ann Transl Med 2021;9(9):802. doi: 10.21037/atm-21-1873

\section{Du et al. LXA4 and its analog BML-111 inhibit melanoma invasion}

and reactive oxygen species production by modulating neutrophil-platelet interaction and CD11b expression. Infect Immun 2011;79:1489-97.

20. Nascimento-Silva V, Arruda MA, Barja-Fidalgo C, et al. Aspirin-triggered lipoxin A4 blocks reactive oxygen species generation in endothelial cells: a novel antioxi-dative mechanism. Thromb Haemost 2007;97:88-98.

21. Wu Y, Zhai H, Wang Y, et al. Aspirin-triggered lipoxin $\mathrm{A}_{4}$ attenuates lipopolysac-charide-induced intracellular ROS in BV2 microglia cells by inhibiting the function of NADPH oxidase. Neurochem Res 2012;37:1690-6.

22. Vieira AM, Neto EH, Figueiredo CC, et al. ATL1, a synthetic analog of lipoxin, modulates endothelial permeability and interaction with tumor cells through a VEGF-dependent mechanism. Biochem Pharmacol 2014;90:388-96.

(English Language Editor: J. Reynolds) 